\title{
Effects of sex and joint action on voluntary activation
}

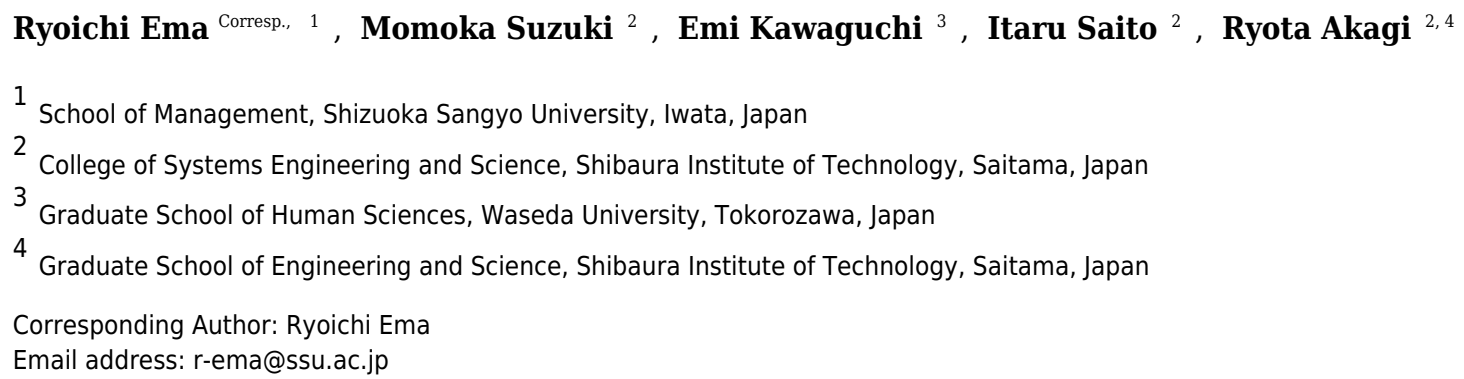

The current study tested the hypothesis that voluntary activation during maximal voluntary contraction (MVC) conditionally depends on sex and joint action. Twenty-eight healthy adults (14 of each sex) performed knee extensor MVC and plantar flexor MVC at extended and flexed knee positions. Voluntary activation during MVC was assessed using a twitch interpolation technique. The voluntary activation during plantar flexor MVC at the extended knee position was significantly lower $(P=0.020,95 \%$ confidence interval 1.4 to 14.6 , Cohen's d for between-subject design $=0.94)$ in women $(88.3 \% \pm 10.0 \%)$ than in men $(96.2 \% \pm 6.6 \%)$. In contrast, no significant sex differences were shown in the voluntary activation during knee extensor MVC (93.7\% $\pm 5.9 \%$ [women] vs. $95.0 \% \pm 3.9 \%$ [men]) and during plantar flexor MVC at the flexed knee position $(90.4 \% \pm 12.2 \%$ [women] vs. $96.8 \% \pm 5.6 \%$ [men]). The voluntary activation during knee extensor MVC was significantly higher $(P=0.001,95 \%$ confidence interval 2.1 to 8.8 , Cohen's $d$ for withinsubject design $=0.69$ ) than that during plantar flexor MVC at the extended knee position in women, whereas the corresponding difference was not observed in men. The results revealed that the existence of sex difference in the voluntary activation during MVC depends on joint action and joint angle . 


\section{Effects of sex and joint action on voluntary activation}

2

3 Ryoichi Ema ${ }^{1}$, Momoka Suzuki $^{2}$, Emi Kawaguchi ${ }^{3}$, Itaru Saito ${ }^{2}$, Ryota Akagi ${ }^{2,4}$

4 1: School of Management, Shizuoka Sangyo University, Iwata, Japan

5 2: College of Systems Engineering and Science, Shibaura Institute of Technology, Saitama, Japan

6 3: Graduate School of Human Sciences, Waseda University, Tokorozawa, Japan

7 4: Graduate School of Engineering and Science, Shibaura Institute of Technology, Saitama, Japan

8

9 Corresponding author: Ryoichi Ema, Ph.D. E-mail: r-ema@ssu.ac.jp 
10 Abstract

11 The current study tested the hypothesis that voluntary activation during maximal voluntary

12 contraction (MVC) conditionally depends on sex and joint action. Twenty-eight healthy adults (14

13 of each sex) performed knee extensor MVC and plantar flexor MVC at extended and flexed knee

14 positions. Voluntary activation during MVC was assessed using a twitch interpolation technique.

15 The voluntary activation during plantar flexor $\mathrm{MVC}$ at the extended knee position was

16 significantly lower $(P=0.020,95 \%$ confidence interval 1.4 to 14.6 , Cohen's $\mathrm{d}$ for between-subject

17 design $=0.94)$ in women $(88.3 \% \pm 10.0 \%)$ than in men $(96.2 \% \pm 6.6 \%)$. In contrast, no significant

18 sex differences were shown in the voluntary activation during knee extensor MVC $(93.7 \% \pm 5.9 \%$

19 [women] vs. $95.0 \% \pm 3.9 \%[\mathrm{men}])$ and during plantar flexor MVC at the flexed knee position

$20(90.4 \% \pm 12.2 \%$ [women] vs. $96.8 \% \pm 5.6 \%[\mathrm{men}])$. The voluntary activation during knee extensor

21 MVC was significantly higher $(P=0.001,95 \%$ confidence interval 2.1 to 8.8 , Cohen's $\mathrm{d}$ for

22 within-subject design $=0.69)$ than that during plantar flexor MVC at the extended knee position

23 in women, whereas the corresponding difference was not observed in men. The results revealed

24 that the existence of sex difference in the voluntary activation during MVC depends on joint action 25 and joint angle. 


\section{Introduction}

A magnitude of muscle activation during maximal voluntary isometric contractions (MVCs) is the major determinant of generated muscular force. Voluntary activation (VA\%), determined by the twitch interpolation technique (Shield \& Zhou, 2004), is an index often used to represent the magnitude of muscle activation.

It has been shown that the magnitude of $\mathrm{VA} \%$ depends on movements at joints (joint action). For example, male participants demonstrated a lower VA\% during knee extensor MVC than during plantar flexor MVC (Behm et al., 2002). As a possible reason for the difference, Behm et al. (2002) proposed the difference in the muscle fiber type compositions of agonist muscle groups, i.e., a difficulty in full recruitment of motor units during knee extensor MVC due to the relatively higher proportion of type II fibers of the quadriceps femoris compared with the triceps surae. The proportion of type II fibers of the vastus lateralis, the largest muscle among the quadriceps femoris in both sexes (Ema et al., 2017), is lower in women than in men (Hunter, 2014). Although the association between $\mathrm{VA} \%$ and muscle fiber type composition is unclear, if the proposal by Behm et al. (2002) is correct, women may show a higher magnitude of VA\% during knee extensor MVC than men. In contrast, the soleus, which has the largest physiological crosssectional area among the triceps surae (Fukunaga et al., 1992), comprises mainly type I fibers even in men (Johnson et al., 1973), suggesting that any possible sex difference of the soleus fiber type composition will be smaller compared with the corresponding difference of the vastus lateralis. Moreover, given that knee flexion reduces the neural and mechanical contribution of the gastrocnemius to plantar flexion strength (Wakahara et al., 2007), it can be assumed that the sex difference in VA\% during plantar flexor MVC, if any, will be small, especially at a flexed knee position. A training program induced a similar extent of strength gains between sexes despite a 
49

smaller magnitude of muscle hypertrophy in women (Lemmer et al., 2000; Melnyk et al., 2009), which may indicate greater neural adaptation by the training in women than in men. Considering that the magnitude of VA\% before training intervention (Gondin et al., 2005) and its traininginduced change (Ema et al., 2018) were related to strength gain, an investigation of the above notions should promote better understanding of the underpinning mechanisms of training-induced strength improvement in men and women.

To the best of our knowledge, no studies have investigated the effects of sex and joint action on VA\% during MVC simultaneously. In the current study, we determined VA\% during knee extensor MVC and plantar flexor MVC at extended and flexed knee positions in both sexes. We tested the hypothesis that VA\% during MVC conditionally depends on sex and joint action.

\section{Methods}

\section{Participants}

A sample size estimation (G*Power 3.1.7, Kiel University, Germany) was performed to detect a within-between interaction for VA\%. The expected effect size, $\alpha$, power, and correlation among repeated measures were set at $0.25,0.05,0.80$, and 0.5 , respectively. The estimation showed that 28 participants are required. It was proposed that physical activity and the existence of practice for strength testing affect the magnitude of VA\% (Hunter et al., 2016). Therefore, we recruited untrained healthy young adults, and all participants visited our laboratory in advance for familiarization and practice in performing MVCs with the experimental setting for the right leg. A total of 28 adults (14 of each sex) with no habitual resistance exercises, knee or ankle injuries participated in the study (Table 1). We confirmed no significant sex difference in the magnitude of habitual physical activity, assessed with the long version of the International Physical Activity 
72 Questionnaire (Craig et al., 2003). The strength testing for knee extension and plantar flexion was

73 performed on different days in random order among the participants. This study was approved by the Ethics Committee of the Shibaura Institute of Technology (Acceptance number: 16-008). All participants were informed of potential risks and the study's purpose, and they provided written informed consent before participation.

\section{Evoked twitch responses}

To provide insights into the effects of muscle fiber type composition on VA\%, we investigated the twitch contractile properties, because the properties have been reported to be associated with the composition (Harridge et al., 1996; Hamada et al., 2000). Participants sat (for knee extensions) or lay supine (for plantar flexions) on the bench of an isokinetic dynamometer (CON-TREX MJ, PHYSIOMED, Germany) while being secured at the pelvis and torso to the dynamometer with nonelastic straps (Figure 1). The knee and hip joint angles were set at $90^{\circ}$ and $80^{\circ}$, respectively, for knee extension (anatomical position $=0^{\circ}$ ). For plantar flexion, the knee joint angle was $0^{\circ}(\mathrm{K} 0)$ or $90^{\circ}(\mathrm{K} 90)$ and the ankle joint angle was $0^{\circ}$ (Kennedy \& Cresswell, 2001). The centers of rotation of the dynamometer and the right knee/ankle joints were visually adjusted. Using a constant current variable voltage stimulator (DS7A, Digitimer Ltd, UK), the quadriceps femoris and triceps surae twitch responses were obtained with rectangular pulses of $1 \mathrm{~ms}$. For the quadriceps femoris, to percutaneously stimulate the femoral nerve, a cathode $(2 \times 2 \mathrm{~cm})$ was placed in the femoral triangle, and an anode $(4 \times 5 \mathrm{~cm})$ was placed in midway between the superior aspect of the greater trochanter and the inferior border of the iliac crest. For the triceps surae, the tibial nerve was stimulated percutaneously in the popliteal fossa with the cathode and over the ventral aspect of the thigh with the anode. The supramaximal stimulus intensity was determined by increasing the 
95 current intensity until plateaus in the twitch torque occurred. Thereafter, five supramaximal twitch

96 responses at a higher current $(\geq 20 \%)$ were obtained every 10 seconds. Torque signals were

97 recorded at $4 \mathrm{kHz}$ and stored in a personal computer after A/D conversion (PowerLab16/35,

98 ADInstruments, Australia). After low-pass filtering the signal at $500 \mathrm{~Hz}$, contraction onset was

manually identified as described previously (Ema et al., 2018). A previous study used the time to

peak twitch torque (TPT), i.e., the duration from torque onset to peak twitch torque, as an index of

estimated muscle fiber type composition (Kubo \& Ikebukuro, 2010). Moreover, TPT was associated with muscle fiber type composition (Hamada et al., 2000). However, TPT is possible to depend on the magnitude of the peak value of twitch torque, making it unsuitable for comparisons between sexes and between different joint actions. Therefore, we determined the twitch torque at $50 \mathrm{~ms}$ from torque onset relative to the peak value of twitch torque (normalized Twitch $_{0-50}$ ) (Balshaw et al., 2016), and used this metric as the index of estimated muscle fiber type composition. The data were averaged across five contractions.

\section{VA\% evaluations}

110 After several warm-up contractions involving two maximal MVCs, participants performed knee extensor/plantar flexor MVCs for $3 \mathrm{~s}$ two times. A one-minute rest was provided between contractions. Verbal encouragement was provided during the contractions. When peak torque was visually observed on screen and $2 \mathrm{~s}$ after the MVC, supramaximal triplet stimulations at $100 \mathrm{~Hz}$ were interpolated. If the difference in the peak value of torque before stimulation was above $10 \%$, an additional contraction was requested. The VA\% was calculated as follows: (1 - [superimposed triplet torque/potentiated resting triplet torque]) $\times 100$ (Miyamoto et al., 2012). The mean of the

117 two trials was used for subsequent analyses. 
119 Statistical analyses

120 Statistical analyses were performed using SPSS version 22 (IBM, USA). All data are shown as

121 means \pm standard deviation. The significance level was set at $P<0.05$. A two-way analysis of

122

123

124

125

126

127

128

129

130

131

132

133

134

135

136

137

138

139

variance (ANOVA) with one between-group factor (sex; men and women) and one within-group factor (joint action; knee extension, plantar flexion in $\mathrm{K} 0$ and K90) was conducted on dependent variables. When a significant interaction of sex $\times$ joint action was shown, follow-up ANOVAs with Bonferroni multiple-comparisons were used. To examine the magnitude of the difference in variables, Cohen's d (between- or within-subject designs, [Lakens 2013]) was calculated as an index of effect size (ES), and 95\% confidence interval of the difference were determined. The thresholds for interpretation of ES were $0.20,0.60$, and 1.20 for small, medium, and large (Hopkins et al., 2009). We considered the differences to be substantial if both $\mathrm{ES}>0.60$ and $P<0.05$.

\section{Results}

Figure 2 shows VA\% during MVC. There was a significant sex $\times$ joint-action interaction $(\mathrm{F}[2$, $52]=3.221, P=0.048)$ and significant main effect of $\operatorname{sex}(\mathrm{F}[1,26]=4.27, P=0.049)$ but not of joint action $(\mathrm{F}[2,52]=1.198, P=0.310)$. Regarding the sex difference, $\mathrm{VA} \%$ during plantar flexor MVC in K0 was significantly higher in men $(96.2 \% \pm 6.6 \%)$ than in women $(88.3 \% \pm$ $10.0 \%$ ), whereas no significant sex difference was found for VA\% during knee extensor MVC $(95.0 \% \pm 3.9 \%$ [men] vs. $93.7 \% \pm 5.9 \%$ [women]) or plantar flexor MVC in K90 $(96.8 \% \pm 5.6 \%$ [men] vs. $90.4 \% \pm 12.2 \%$ [women]), with the observed effects as small to medium (Table 2). For joint action dependency, VA\% during knee extensor MVC was significantly higher than that 
140 during plantar flexor MVC in K0 in women, with the effect being medium, but not in men. No

141 corresponding differences were shown between other joint actions in each sex.

The normalized Twitch $_{0-50}$ results are described in Figure 3. A significant main effect of joint action $(\mathrm{F}[2,52]=90.321, P<0.001)$ without a main effect of $\operatorname{sex}(\mathrm{F}[1,26]=0.376, P=$ $0.545)$ or an interaction of the two factors $\left(\mathrm{F}[2,52]=0.607, P=0.549\right.$, partial $\left.\eta^{2}=0.023\right)$ was shown. The normalized Twitch $0-50$ of knee extension was significantly greater than those of plantar flexions for both extended and flexed knee positions, with the effects being large.

\section{Discussion}

The main finding of the current study was that the substantial sex difference in VA\% was shown only during plantar flexor MVC at the extended knee position. Compared with men, women showed lower VA\% during plantar flexor MVC at the extended knee position, and the magnitude of sex difference was interpreted as medium $(\mathrm{ES}=0.94)$. In contrast, a clear sex difference in VA\% was not observed during knee extensor MVC or during plantar flexor MVC at the flexed knee position. In addition, in women but not in men, VA\% during plantar flexor MVC at the extended knee position was substantially different from that during knee extensor MVC. These results indicate that the sex difference in VA\% during MVC depended on joint action.

The substantial sex difference in VA\% was shown only during plantar flexor MVC in K0. The normalized Twitch $_{0-50}$ was not significantly different between sexes for any joint action (Figure 2), suggesting that the muscle fiber type composition is not a major factor for the observed sex and joint-action dependency in VA\%. Co-contraction of the antagonist tibialis anterior during plantar flexor MVC might be the explainable factor for the current result. It was shown that compared with males, females exhibited higher magnitude of the tibialis anterior activation relative 
163 to medial gastrocnemius activation during the push-off phase of countermovement jumping 164 (Márquez et al., 2017). During countermovement jumping, just before take-off, the fascicles of the

165 166

167

medial gastrocnemius have been reported to contract quasi-isometrically (Kurokawa et al., 2003); therefore, the contraction type of the triceps surae may be partly similar between the current (i.e., isometric contraction) and previous (Márquez et al., 2017) studies. Because of reciprocal inhibition (Crone et al., 1987), co-contraction of the tibialis anterior during plantar flexor MVC could diminish the magnitude of triceps surae activation during plantar flexion. In contrast, the absence of a clear sex difference in VA\% during knee extensor MVC (Figure 2) is in line with previous studies (Krishnan \& Williams, 2009; Lee et al., 2017), and Krishnan \& Williams (2009) observed that the magnitude of hamstring activation as antagonists during knee extensor MVC was not different between sexes. Such notions may be also related to the substantially higher VA\% during knee extensor MVC than during plantar flexor MVC in $\mathrm{K} 0$ only in women. Taken together, a possible sex difference in the antagonist activation may account for the current sex- and jointaction differences in VA\%.

The lack of significant difference in VA\% between joint actions in male participants is not consistent with the previous finding of a higher value of VA $\%$ during plantar flexor MVC than during knee extensor MVC in men (Behm et al., 2002). This discrepancy may be related to the difference in participant backgrounds. We recruited untrained participants because resistance training can affect the magnitude of VA\% (Ema et al., 2018). In contrast, Behm et al. (2002) examined subjects who participated in habitual resistance exercises or competitive sport activities. Although the kind of resistance training that the subjects had performed was not mentioned, it is possible that the effects of training on VA\% during MVC differed between joint actions, likely 
185 186 188 189

resulting in the higher VA\% during plantar flexor MVC than during knee extensor MVC (Behm et al., 2002).

Our findings may provide some implication regarding the training-induced strength gains. Previous studies demonstrated that greater muscle hypertrophy in men than in women after resistance training were accompanied by a lack of sex difference in knee extension strength gains (Lemmer et al., 2000; Melnyk et al., 2009). The previous results imply a greater neural adaptation in women than in men, because there was a negative correlation between VA\% before training and the magnitude of strength improvement (Gondin et al., 2005). However, no significant sex difference in VA\% during knee extensor MVC was found in the present study; therefore, it is difficult to explain the aforementioned results in terms of a sex difference in neural adaptations. In contrast, our data might suggest the greater training-induced increase in plantar flexion strength in women than in men, and the greater strength gains in plantar flexion than in knee extension in women; future attempts are required to clarify this subject.

\section{Conclusion}

The substantial sex difference in VA\% was limited during plantar flexor MVC at the extended knee position, and only women showed joint action dependency in VA\%. These results revealed that the existence of sex difference in VA\% during MVC depends on joint action and joint angle. 


\section{References}

Balshaw TG, Massey GJ, Maden-Wilkinson TM, Tillin NA, Folland JP. Training-specific functional, neural, and hypertrophic adaptations to explosive- vs. sustained-contraction strength training. J Appl Physiol (2016); 120: 1364-1373.

Behm DG, Whittle J, Button D, Power K. Intermuscle differences in activation. Muscle Nerve (2002); 25: 236-243.

Craig CL, Marshall AL, Sjöström M, Bauman AE, Booth ML, Ainsworth BE, Pratt M, Ekelund U, Yngve A, Sallis JF, Oja P. International physical activity questionnaire: 12-country reliability and validity. Med Sci Sports Exerc (2003); 35: 1381-1395.

Crone C, Hultborn H, Jespersen B, Nielsen J. Reciprocal Ia inhibition between ankle flexors and extensors in man. J Physiol (1987); 389: 163-185.

Ema R, Wakahara T, Hirayama K, Kawakami Y. Effect of knee alignment on the quadriceps femoris muscularity: Cross-sectional comparison of trained versus untrained individuals in both sexes. PLoS One (2017); 12: e0183148.

Ema R, Saito I, Akagi R. Neuromuscular adaptations induced by adjacent joint training. Scand $J$ Med Sci Sports (2018); 28: 947-960.

Fukunaga T, Roy RR, Shellock FG, Hodgson JA, Day MK, Lee PL, Kwong-Fu H, Edgerton VR. Physiological cross-sectional area of human leg muscles based on magnetic resonance imaging. J Orthop Res (1992); 10: 928-934.

Gondin J, Guette M, Ballay Y, Martin A. Electromyostimulation training effects on neural drive and muscle architecture. Med Sci Sports Exerc (2005); 37: 1291-1299.

Hamada T, Sale DG, MacDougall JD, Tarnopolsky MA. Postactivation potentiation, fiber type, and twitch contraction time in human knee extensor muscles. J Appl Physiol (2000); 88: 2131- 
2137.

227

228

229

230

231

232

233

234

235

236

237

238

239

240

241

242

243

244

245

246

247

248

Harridge SD, Bottinelli R, Canepari M, Pellegrino MA, Reggiani C, Esbjörnsson M, Saltin B. Whole-muscle and single-fibre contractile properties and myosin heavy chain isoforms in humans. Pflugers Arch (1996); 432: 913-920.

Hopkins WG, Marshall SW, Batterham AM, Hanin J. Progressive statistics for studies in sports medicine and exercise science. Med Sci Sports Exerc. (2009); 41: 3-13.

Hunter SK. Sex differences in human fatigability: mechanisms and insight to physiological responses. Acta Physiol (2014); 210: 768-789.

Hunter SK, Pereira HM, Keenan KG. The aging neuromuscular system and motor performance. $J$ Appl Physiol (2016); 121: 982-995.

Johnson MA, Polgar J, Weightman D, Appleton D. Data on the distribution of fibre types in thirtysix human muscles. An autopsy study. J Neurol Sci (1973); 18: 111-129.

Kennedy PM, Cresswell AG. The effect of muscle length on motor-unit recruitment during isometric plantar flexion in humans. Exp Brain Res (2001); 137: 58-64.

Krishnan C, Williams GN. Sex differences in quadriceps and hamstrings EMG-moment relationships. Med Sci Sports Exerc (2009); 41: 1652-1660.

Kubo K, Ikebukuro T. Relationship between muscle fiber type and tendon properties in young males. Muscle Nerve (2010); 42: 127-129.

Kurokawa S, Fukunaga T, Nagano A, Fukashiro S. Interaction between fascicles and tendinous structures during counter movement jumping investigated in vivo. J Appl Physiol (2003); 95: $2306-2314$.

Lakens D. Calculating and reporting effect sizes to facilitate cumulative science: a practical primer for t-tests and ANOVAs. Front Psychol (2013); 4: 863. 
249 Lee A, Baxter J, Eischer C, Gage M, Hunter S, Yoon T. Sex differences in neuromuscular function 250 after repeated eccentric contractions of the knee extensor muscles. Eur J Appl Physiol (2017); 117: 1119-1130.

252

253

254

255

256

257

258

259

260

261

262

263

264

265

266

Lemmer JT, Hurlbut DE, Martel GF, Tracy BL, Ivey FM, Metter EJ, Fozard JL, Fleg JL, Hurley BF. Age and gender responses to strength training and detraining. Med Sci Sports Exerc (2000); 32: 1505-1512.

Márquez G, Alegre LM, Jaén D, Martin-Casado L, Aguado X. Sex differences in kinetic and neuromuscular control during jumping and landing. J Musculoskelet Neuronal Interact (2017); 17: 409-416.

Melnyk JA, Rogers MA, Hurley BF. Effects of strength training and detraining on regional muscle in young and older men and women. Eur J Appl Physiol (2009); 105: 929-938.

Miyamoto N, Fukutani A, Yanai T, Kawakami Y. Twitch potentiation after voluntary contraction and neuromuscular electrical stimulation at various frequencies in human quadriceps femoris. Muscle Nerve (2012); 45: 110-115.

Shield A, Zhou S. Assessing voluntary muscle activation with the twitch interpolation technique. Sports Med (2004); 34: 253-267.

Wakahara T, Kanehisa H, Kawakami Y, Fukunaga T. Fascicle behavior of medial gastrocnemius muscle in extended and flexed knee positions. J Biomech (2007); 40: 2291-2298. 


\section{Figure Captions}

\section{Figure 1}

269 Schematic illustration of the experimental setup for knee extension (A), plantar flexion at extended 270 (B) and at flexed knee positions (C).

Figure 2

273 Scatterplots showing individual data of voluntary activation (VA\%) during maximal voluntary 274 contractions of knee extension, plantar flexion at extended (K0) and at flexed (K90) knee positions. 275 Solid line shows the mean value. *Indicates a significant difference between joint actions. †Shows 276 a significant difference between sexes.

\section{Figure 3}

279 Scatterplots showing individual data of twitch torque at $50 \mathrm{~ms}$ from torque onset relative to the 280 peak value of twitch torque (Normalized twitch ${ }_{0-50}$ ) of knee extension, plantar flexion at extended 281 (K0) and at flexed (K90) knee positions. Solid line shows the mean value. *Indicates a significant 282 difference between joint actions. 


\section{Table $\mathbf{1}$ (on next page)}

Physical characteristics of participants.

MET, metabolic equivalent. Data are shown as mean \pm standard deviation. 
Table 1 Physical characteristics of participants

\begin{tabular}{|c|c|c|c|c|c|c|c|c|}
\hline \multirow{2}{*}{$\square$} & \multirow{2}{*}{ 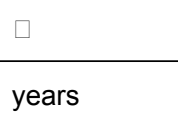 } & \multicolumn{3}{|c|}{ Men $(n=14)$} & \multirow[t]{2}{*}{$\square$} & \multicolumn{3}{|c|}{ Women $(n=14)$} \\
\hline & & 23 & \pm & 4 & & 22 & \pm & 1 \\
\hline Height & $\mathrm{cm}$ & 169.7 & \pm & 4.4 & & 157.6 & \pm & 4.1 \\
\hline Body mass & $\mathrm{kg}$ & 62.0 & \pm & 6.2 & & 51.5 & \pm & 6.6 \\
\hline Physical activity & MET $\min / \mathrm{wk}$ & 3108 & \pm & 2177 & $\square$ & 2836 & \pm & 2542 \\
\hline
\end{tabular}

$\mathrm{MET}$, metabolic equivalent. Data are shown as mean \pm standard deviation. 


\section{Table 2 (on next page)}

Statistical results of the difference between sexes and between joint actions.

ES, Cohen's d for between- or within-subject designs; KE, knee extension; Normalized twitch $_{0-50}$, twitch torque at $50 \mathrm{~ms}$ from torque onset relative to the peak value of twitch torque; PF in K0, plantar flexion at extended knee position; PF in K90, plantar flexion at flexed knee position; VA\%, voluntary activation. 
Table 2. Statistical results of the difference between sexes and between joint actions.

\begin{tabular}{llrr}
\hline & P value & ES & $\begin{array}{c}95 \% \text { confidence } \\
\text { interval }\end{array}$ \\
\hline \hline VA\% & & & \\
Males vs. Females & & & \\
KE & 0.501 & 0.26 & -2.6 to 5.1 \\
PF in K0 & 0.020 & 0.94 & 1.4 to 14.6 \\
PF in K90 & 0.086 & 0.68 & -1.0 to 13.8 \\
KE vs. PF in K0 & & & \\
Males & 0.421 & 0.24 & -4.4 to 1.9 \\
Females & 0.001 & 0.69 & 2.1 to 8.8 \\
KE vs. PF in K90 & & & \\
Males & 0.418 & 0.39 & -4.6 to 0.9 \\
Females & 0.152 & 0.36 & -2.9 to 9.5 \\
PF in K0 vs. in K90 & & & \\
Males & 0.772 & 0.10 & -1.9 to 0.7 \\
Females & 0.299 & 0.19 & -8.2 to 3.9
\end{tabular}

Normalized twitch ${ }_{0-50}$

Males vs. Females

$\mathrm{KE}$

$\mathrm{PF}$ in $\mathrm{KO}$

0.545

0.25

-5.6 to 2.8

0.545

0.08

-3.3 to 4.0

$\mathrm{PF}$ in $\mathrm{K} 90$

0.545

0.33

-6.4 to 2.6

$\mathrm{KE}$ vs. $\mathrm{PF}$ in $\mathrm{KO}$

Males

$<0.001$

2.00

8.3 to 15.1

Females

$<0.001$

3.37

10.1 to 16.7

$\mathrm{KE}$ vs. PF in K90

Males

$<0.001$

1.87

8.0 to 16.8

Females

$<0.001$

2.80

8.3 to 15.4

$\mathrm{PF}$ in $\mathrm{KO}$ vs. in $\mathrm{K} 90$

Males

1.000

0.12

-1.4 to 2.7

Females

1.000

0.35

-3.5 to 0.4

ES, Cohen's d for between- or within-subject designs; KE, knee extension; Normalized twitch $0-50$, twitch torque at $50 \mathrm{~ms}$ from torque onset relative to the peak value of twitch torque; PF in K0, plantar flexion at extended knee position; PF in K90, plantar flexion at flexed knee position; VA\%, voluntary activation. 


\section{Figure 1 (on next page)}

Schematic illustration of the experimental setup for knee extension (upper), plantar flexion at extended (middle) and at flexed knee positions (lower). 
A

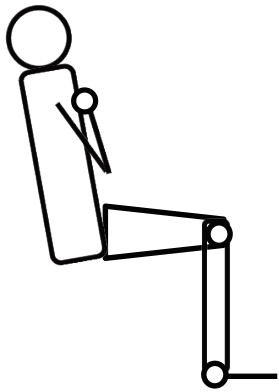

B

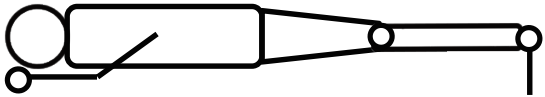

C

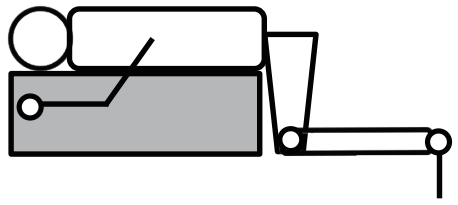




\section{Figure 2 (on next page)}

Scatterplots showing individual data of voluntary activation (VA\%) during maximal voluntary contractions of knee extension, plantar flexion at extended $(\mathrm{K} 0)$ and at flexed (K90) knee positions.

Solid line shows the mean value. *Indicates a significant difference between joint actions. †Shows a significant difference between sexes. 


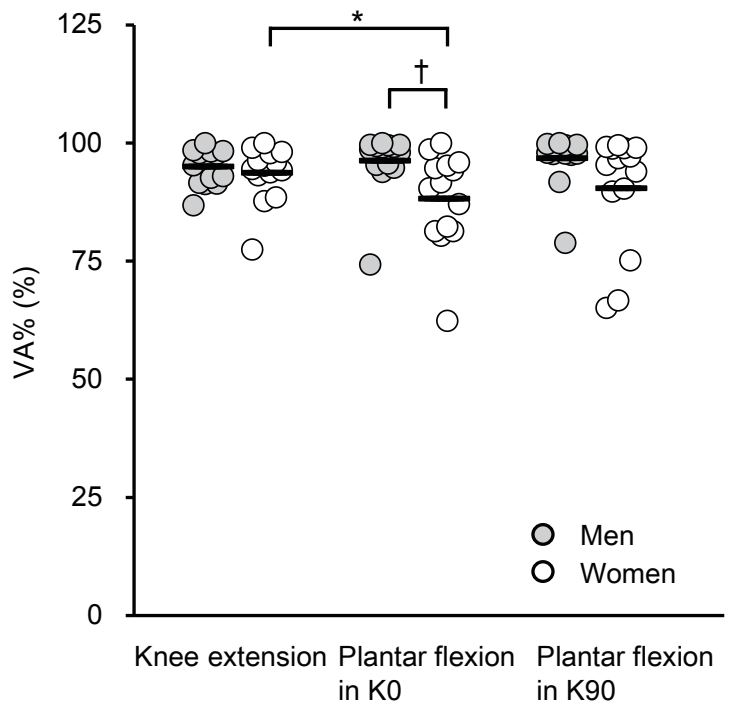




\section{Figure 3 (on next page)}

Scatterplots showing individual data of twitch torque at $50 \mathrm{~ms}$ from torque onset relative to the peak value of twitch torque (Normalized twitch ${ }_{0.50}$ ) of knee extension, plantar flexion at extended (KO) and at flexed (K90) knee positions.

Solid line shows the mean value. *Indicates a significant difference between joint actions. 


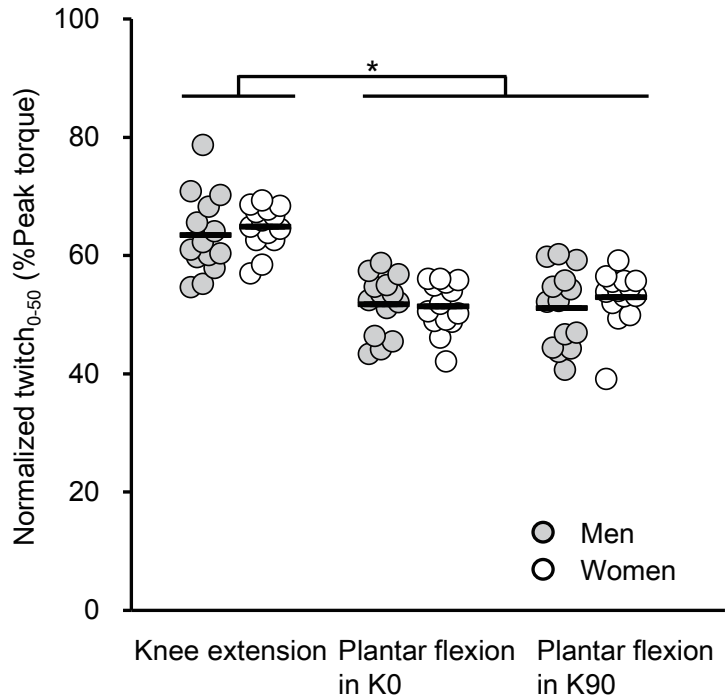

